\title{
Right ventricular involvement in left ventricular non-compaction cardiomyopathy
}

\author{
Simon F. Stämpfli ${ }^{1,2, *}$, Alexander Gotschy ${ }^{1,3} *$, Pascal Kiarostami ${ }^{1}$, Tardu Özkartal ${ }^{1}$, \\ Christiane Gruner ${ }^{1}$, Markus Niemann ${ }^{1,4}$, Robert Manka ${ }^{1,5}$, Felix C. Tanner ${ }^{1}$ \\ ${ }^{1}$ Department of Cardiology, University Heart Center Zurich, Switzerland \\ ${ }^{2}$ Department of Cardiology, Heart Center Lucerne, Luzerner Kantonsspital, Lucerne, Switzerland \\ ${ }^{3}$ Institute for Biomedical Engineering, University and ETH Zurich, Switzerland \\ ${ }^{4}$ Faculty Mechanical and Medical Engineering, Furtwangen University, Germany \\ ${ }^{5}$ Institute of Diagnostic and Interventional Radiology, University Hospital Zurich, Switzerland
}

\begin{abstract}
Background: Left ventricular non-compaction cardiomyopathy (LVNC) features extensive trabeculations. Involvement of the right ventricle $(R V)$ has been reported; however, distinction from normal $R V$ trabeculation is difficult. This study aimed at assessing RV morphology and function in LVNC by cardiac magnetic resonance (CMR) and transthoracic echocardiography (TTE).

Methods: Dimensional and functional parameters were assessed according to guidelines. Novel CMR parameters were RV end-diastolic (ED) trabeculated area, RV ED trabeculated volume, and RV ED non-compacted to compacted (NC/N) ratio in short axis (SAX) as well as in four-chamber view (4CH). Results: Twenty patients with LVNC and 20 controls were included. RV size and function were comparable in LVNC and controls and exhibited a good correlation between TTE and CMR. Although RV trabeculated area, $R V$ trabeculated volume, and $R V E D N C / C$ ratio in $S A X$ as well as in $4 C H$ were larger in $L V N C$, there was a major overlap with values in controls. $R V E D N C / C$ ratio in SAX correlated with $L V E D N C / C$ ratio (not in $4 C H$ ). Quantitative assessment of $R V$ non-compaction was not feasible in TTE.
\end{abstract}

Conclusions: Right ventricle size and function in LVNC can be measured by CMR and TTE, while RV trabeculation can only be quantified by CMR. RV myocardium displays more trabeculations in LVNC; however, overlap with normal individuals is extensive, not allowing separation of patients with LVNC from controls. (Cardiol J 2022; 29, 3: 454-462)

Key words: trabeculation, cardiac magnetic resonance imaging, echocardiography, left ventricular non-compaction cardiomyopathy

\section{Introduction}

Left ventricular non-compaction cardiomyopathy (LVNC) is characterized by a two-layered myocardium involving a thin, compacted, outer layer and a thick, non-compacted, inner layer with deep recesses between prominent trabeculations [1]. During recent years, the awareness of LVNC has increased [2-6], with wider recognition of the disease and systematic family screening, the number of symptomatic and asymptomatic patients diagnosed with LVNC is growing [7-14].

While most studies have focused on the left ventricle (LV) [15-17], prominent trabeculation has also been reported in the right ventricle (RV) of patients with LVNC $[5,18,19]$. In addition, RV

Address for correspondence: Felix C. Tanner, MD, Department of Cardiology, University Heart Center Zurich, Rämistrasse 100, CH-8091 Zurich, Switzerland, tel: +41 4425587 00, fax +4144255 87 01, e-mail: felix.tanner@usz.ch

Received: 20.03.2020 Accepted: 29.06.2020 Early publication date: 10.07.2020

*Simon F. Stämpfli and Alexander Gotschy share first authorship.

This article is available in open access under Creative Common Attribution-Non-Commercial-No Derivatives 4.0 International (CC BY-NC-ND 4.0) license, allowing to download articles and share them with others as long as they credit the authors and the publisher, but without permission to change them in any way or use them commercially. 
systolic function was decreased in patients with advanced LVNC and seemed to be associated with impaired outcome [20-22]. However, the extent and incidence of RV involvement remains unclear. Cardiac magnetic resonance (CMR) performed in 56 LVNC patients revealed in only 6 patients RV non-compaction as defined somewhat arbitrarily by the presence of recesses within the inflow area of the RV involving at least $75 \%$ of the RV thickness [21]. RV apical trabecular thickness assessed by CMR correlated with the extent of LV involvement in LVNC, whereas RV end-diastolic (ED) non-compacted to compacted $(\mathrm{NC} / \mathrm{C})$ ratio in four-chamber view $(4 \mathrm{CH})$ did not differ from normal ventricles [22]. RV systolic function was decreased in patients with advanced LVNC and seemed to be associated with enhanced RV trabeculation and impaired outcome [20-23].

Due to its complex shape, comprehensive evaluation of the RV is difficult by transthoracic two-dimensional echocardiography (TTE) [20]. In addition, the RV exhibits a substantially higher number of trabeculations than the LV even in healthy individuals, wherefore it is challenging to differentiate between normal and pathologic anatomy in patients with suspected LVNC [22]. CMR provides a full volume three-dimensional (3D) dataset independent of acoustic windows and is regarded as the reference method for assessing the RV in various cardiac diseases [24]. It is of great value for diagnosis and morphological description of LVNC in the LV and indeed has become one of the standard modalities for assessing LVNC patients [25-28]. On the other hand, due to its wide availability and high versatility, echocardiography is still the standard tool for assessment of LV and $\mathrm{RV}$ in patients with cardiomyopathies.

Neither the optimal imaging modality nor standardized measurements for RV assessment in LVNC patients have been defined. This study aims at assessing RV morphology in LVNC patients versus controls by introducing novel CMR parameters such as trabeculated area, trabeculated volume, and $\mathrm{NC} / \mathrm{C}$ ratio in short axis (SAX) and at comparing the suitability of CMR and TTE for diagnosing RV involvement in LVNC patients.

\section{Methods}

Twenty patients with LVNC (fulfilling both TTE [15] and CMR criteria [27]) and 20 healthy controls (age and gender matched) underwent TTE and CMR at the University Hospital Zurich between 2011 and 2016. Measurements were per- formed in a blinded manner. Patient records were reviewed for baseline characteristics, New York Heart Association (NYHA) functional class, body height, body weight, blood pressure, heart rate, and medication. The study was approved by the local ethical committee.

All CMR exams were performed on a clinical 1.5 T scanner (Achieva, Philips Healthcare, Best, The Netherlands) using a 5-channel cardiac coil array. Steady-state free precession cine images (echo time/repetition time $1.6 / 3.3 \mathrm{~ms}$, flip angle $60^{\circ}$ ) were acquired in three long-axis views (2-, 3-, and 4-chamber view) and a stack of short-axis slices covering the whole LV and RV. A single reader performed all CMR analysis in a blinded manner using GTVolume software (GyroTools LLC, Zurich, Switzerland). Commonly measured dimensional and functional parameters were assessed according to current guidelines and recommendations [29, 30]. As novel CMR parameters RV ED trabeculated area in $4 \mathrm{CH}$ view, RV ED trabeculated volume, and $\mathrm{RV} \mathrm{ED} \mathrm{NC/N}$ ratio in short axis (SAX) and long axis (LAX) were introduced (Fig. 1A-E). RV ED trabeculated area was quantified by manually contouring the trabeculation in $4 \mathrm{CH}$ view, while $\mathrm{RV}$ ED trabeculated volume was assessed by summation of the trabeculated area in all RV short axes multiplied by the slice thickness. RV ED NC/C values in long and short axis are reported as the maximal ratio of the thickness of $\mathrm{NC}$ to $\mathrm{C}$ layer measured at a single location perpendicular to the compacted wall.

Echocardiographic studies were performed on commercially available echocardiography units (GE E95 and E9, GE Healthcare, Horten, Norway and Philips iE33 and Epic, Philips Medical Systems, Erlangen, Germany) equipped with multi-frequency transducers (1.5-4 MHz). All examinations were performed by experienced sonographers and stored on a digital workstation for subsequent off-line analysis (Xcelera R4.1, Philips Medical Systems, Erlangen, Germany). A modified apical $4 \mathrm{CH}$ view focusing on the RV was used to measure RV area and fractional area change (FAC) by tracing the endocardial surface of the RV compacted myocardial layer both in systole and diastole [31]. Commonly measured dimensional and functional parameters were assessed according to current guidelines and recommendations [32].

\section{Statistical analysis}

Statistical analysis was performed using GraphPad Prism (Version 5.04, La Jolla, USA). Normal distribution of data was confirmed using the 


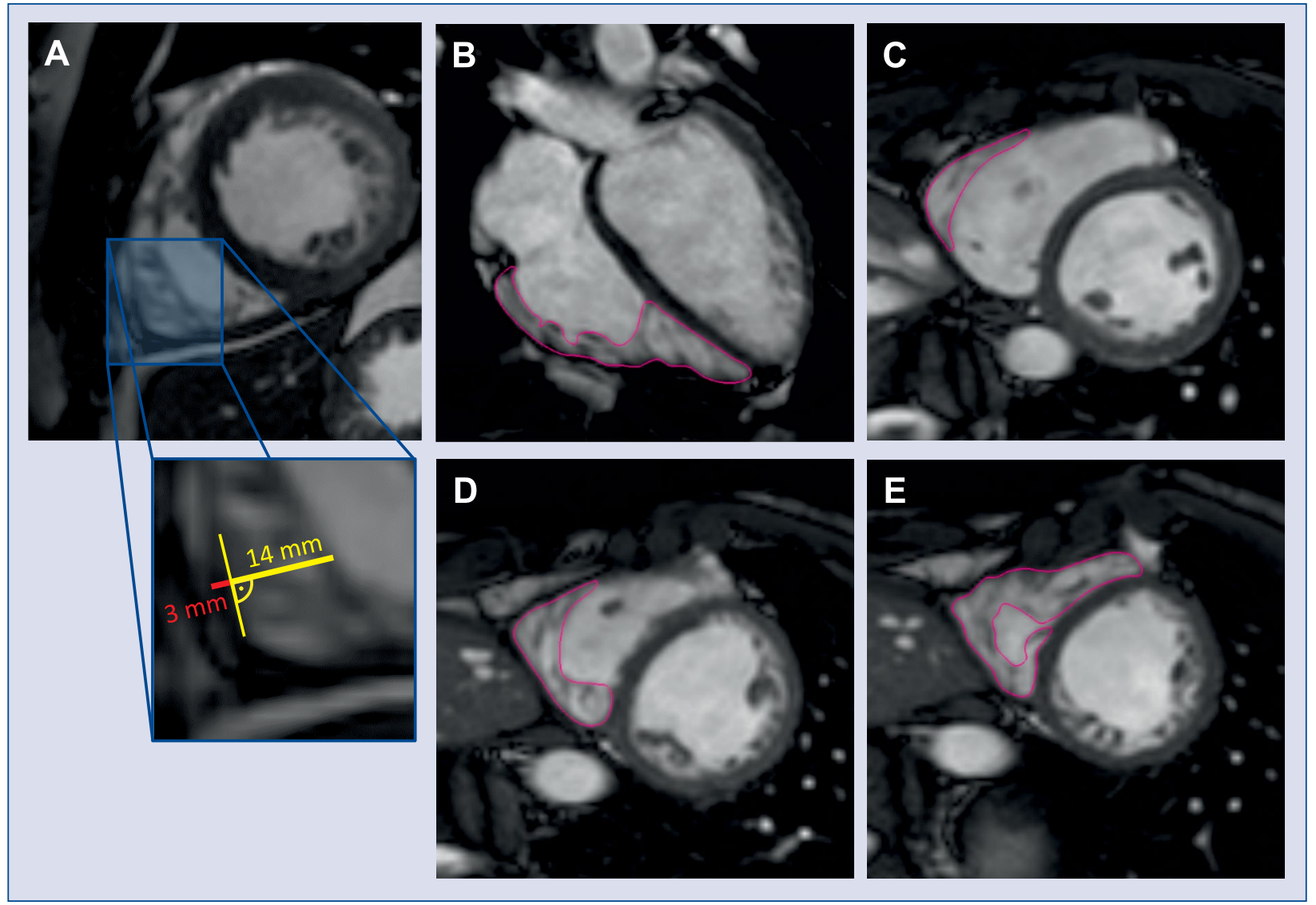

Figure 1. Example of right ventricular end-diastolic non-compacted to compacted ratio as assessed in short axis view (A), right ventricular end-diastolic trabeculated area assessed in four-chamber view (B), and right ventricular end-diastolic trabeculated volume as assessed in short-axis views on a basal (C), midventricular (D) and apical level (E).

Shapiro-Wilk test. Normally distributed continuous values are depicted as mean \pm standard deviation (SD), non-normally distributed continuous data as median and interquartile range (IQR). Categorical data is displayed as number (percentage). To determine the statistical significance between LVNC and control group, the unpaired Student t-test was used. The agreement between CMR and echocardiography measurements was assessed with the Spearman rank correlation. A p-value $<0.05$ was considered statistically significant.

\section{Results}

Twenty patients (9 females, $45 \%$ ) with confirmed LVNC and 20 healthy subjects ( 7 females, $35 \%$ ) were included in the study. The median age of patients with LVNC at the time of CMR was 46 (IQR 33-57) years, that of controls was 54 (IQR $43-57)$ years $(p=0.16)$. In 29 subjects, TTE and CMR took place on the same day; in the remaining 11 subjects, the median time between the two examinations was 11 (4-40) days. CMR-based LV ejection fraction (LVEF) was lower in LVNC patients as compared to controls (52\% vs. $62 \%$, $\mathrm{p}<0.0001)$. However, median LVEF in LVNC patients was only mildly impaired (median $53 \%$, IQR 48-57\%); 8 (40\%) patients had a normal LVEF $(\geq 55 \%)$. Heart rate was slightly higher and systolic blood pressure lower in LVNC patients as compared to controls. Table 1 summarizes baseline characteristics.

Right ventricular end-diastolic area exhibited good correlation between TTE and CMR (Fig. 2, absolute values Table 2$)$ in $\operatorname{LVNC}(\mathrm{r}=0.66, \beta=0.85$, $\mathrm{p}=0.0016)$ and in controls $(\mathrm{r}=0.88, \beta=0.80$, $\mathrm{p}<0.0001)$. RV ED area was higher in CMR as compared to TTE for LVNC (agreement of $\triangle \mathrm{CMR}$-TTE $=$ $=7.5 \mathrm{~cm}^{2}, 95 \%$ limits of agreement $0.007-15.1$ ) and for controls (agreement of $\Delta$ CMR-TTE $=7.8 \mathrm{~cm}^{2}$, 95\% limits of agreement 1.8-13.7). Similarly, RV ED basal diameter was higher in CMR as compared to TTE for both groups (LVNC: agreement of $\Delta$ CMR-TTE $=7.9 \mathrm{~mm}, 95 \%$ limits of agreement 
Table 1. Patient characteristics.

\begin{tabular}{|c|c|c|c|}
\hline Characteristic & LVNC (n = 20) & Controls $(n=20)$ & $\mathbf{P}$ \\
\hline Age [years] & $46(33-57)$ & $54(43-57)$ & 0.16 \\
\hline Female sex & $9(45 \%)$ & $7(35 \%)$ & 0.52 \\
\hline Body mass index $\left[\mathrm{kg} / \mathrm{m}^{2}\right]$ & $24.3 \pm 4.0$ & $26.2 \pm 4.6$ & 0.09 \\
\hline Heart rate $[\mathrm{bpm}]$ & $65.5 \pm 11.6$ & $64.8 \pm 13.1$ & 0.6 \\
\hline Systolic blood pressure [mmHg] & $117 \pm 13.4$ & $131 \pm 9.7$ & 0.03 \\
\hline Diastolic blood pressure $[\mathrm{mmHg}]$ & $71.0 \pm 8.5$ & $78.6 \pm 8.8$ & 0.07 \\
\hline \multicolumn{4}{|l|}{ NYHA class: } \\
\hline Class I & $17(85 \%)$ & $20(100 \%)$ & \\
\hline Class II & $3(15 \%)$ & $0(0 \%)$ & \\
\hline Left ventricular ejection fraction (CMR) [\%] & $51.6 \pm 8.6$ & $62.0 \pm 4.3$ & $<0.0001$ \\
\hline \multicolumn{4}{|l|}{ Medication: } \\
\hline Acetylsalicylic acid & $1(5 \%)$ & $0(0 \%)$ & \\
\hline Phenprocoumon & $3(15 \%)$ & $0(0 \%)$ & \\
\hline Beta-blocker & $5(25 \%)$ & $0(0 \%)$ & \\
\hline ACEI or ARB & $8(40 \%)$ & $0(0 \%)$ & \\
\hline Diuretics & $6(30 \%)$ & $0(0 \%)$ & \\
\hline
\end{tabular}

Data are shown as mean \pm standard deviation or median (interquartile range) or number (percentage). ACEI - angiotensin-converting enzyme inhibitor; ARB - angiotensin-receptor blocker; CMR — cardiac magnetic resonance imaging; LVNC - left ventricular non-compaction cardiomyopathy; NYHA - New York Heart Association

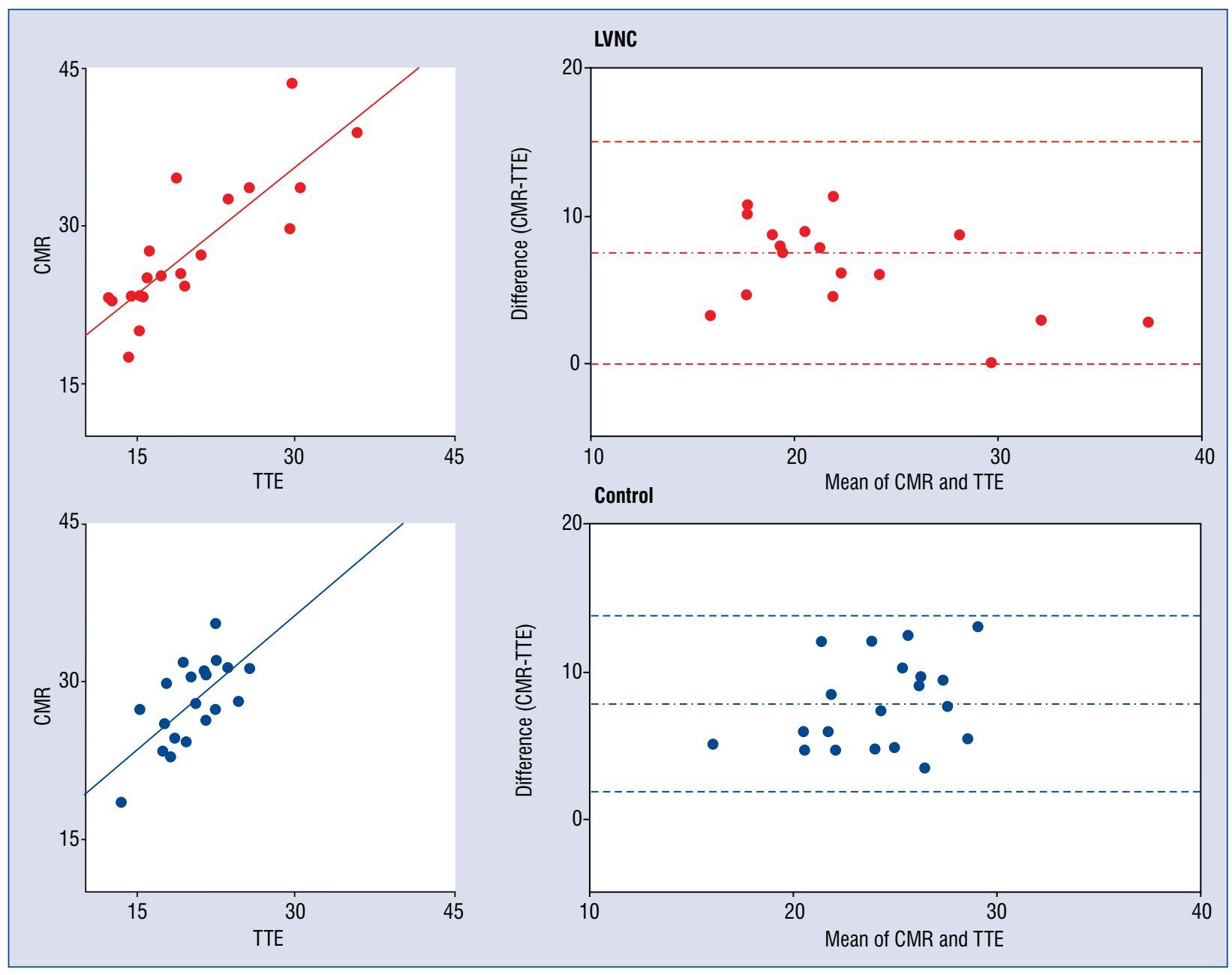

Figure 2. Right ventricular end-diastolic area as measured in transthoracic echocardiography (TTE) and cardiac magnetic resonance (CMR) exhibits good correlation between the two methods in both left ventricular non-compaction cardiomyopathy (LVNC) patients and controls. Scatter plot correlation graph (left), Bland-Altman plot (right). 
Table 2. Structural and functional right ventricular parameters in transthoracic echocardiography (TTE) and cardiac magnetic resonance imaging (CMR).

\begin{tabular}{lccc}
\hline Parameter & LVNC $(\mathbf{n}=\mathbf{2 0})$ & Controls $(\mathbf{n}=20)$ & $\mathbf{P}$ \\
\hline RV ED area $\left[\mathrm{cm}^{2}\right]$ & & & \\
CMR & $25.3(23.2-33.3)$ & $28.0(25.0-31.2)$ & 0.86 \\
TTE & $18.1(15.3-25.2)$ & $20.4(17.9-22.5)$ & 0.96 \\
RV ED basal diameter [mm]: & & & 0.18 \\
CMR & $36(30-41)$ & $41(37-44)$ & 0.4 \\
TTE & $28(25-32)$ & $30(27-32)$ & 0.25 \\
FAC [\%]: & & $42.8 \pm 6.2$ & 0.62 \\
CMR & $46.3 \pm 11.8$ & $41.8 \pm 5.0$ & \\
TTE & $43.0 \pm 9.6$ & & \\
\hline
\end{tabular}

Data are shown as mean \pm standard deviation or median (interquartile range). ED — end-diastolic; FAC — fractional area change; LVNC — left ventricular non-compaction cardiomyopathy; RV — right ventricle

Table 3. Quantitative assessment of right ventricle (RV) non-compaction in cardiac magnetic resonance imaging (CMR).

\begin{tabular}{lccc}
\hline Parameter & LVNC $(\mathbf{n}=20)$ & Controls $(\mathbf{n}=20)$ & P \\
\hline RV ED trabeculated area $\left[\mathrm{cm}^{2}\right]$ & $9.15 \pm 3.69$ & $6.47 \pm 2.97$ & 0.048 \\
RV ED trabeculated volume $[\mathrm{mL}]$ & $35.2(25.6-61.3)$ & $27.0(19.5-35.2)$ & 0.028 \\
RV ED NC/C ratio in SAX & $3.93(3.39-5.18)$ & $2.96(2.48-3.69)$ & 0.001 \\
RV ED NC/C ratio in 4CH & $3.3(2.94-4.13)$ & $2.73(2.00-3.10)$ & 0.019 \\
\hline
\end{tabular}

Data are shown as mean \pm standard deviation or median (interquartile range). ED — end-diastolic; LVNC — left ventricular non-compaction cardiomyopathy; NC/C - non-compacted to compacted; SAX — short axis; $4 \mathrm{CH}$ - four-chamber view

-2.5-18.4; controls: agreement of $\triangle \mathrm{CMR}-\mathrm{TTE}=$ $=9.7 \mathrm{~mm}, 95 \%$ limits of agreement $-0.9-20.1$ ).

Fractional area change was assessed as a functional parameter and exhibited good correlation between the two imaging modalities in LVNC $(\mathrm{r}=0.72, \beta=0.92, \mathrm{p}=0.0004)$ and in controls $(\mathrm{r}=0.56, \beta=0.65, \mathrm{p}=0.011)$. The bias between the two methods was minimal in LVNC (agreement of $\triangle \mathrm{CMR}-\mathrm{TTE}=3.3 \%, 95 \%$ limits of agreement -12.1-18.7) and in controls (agreement of $\Delta$ CMR-TTE $=1.1 \%, 95 \%$ limits of agreement -9.79-11.93).

In TTE, quantitative assessment of RV noncompaction was not feasible. In particular, the decline of lateral resolution with imaging depth hampered a reliable quantification of RV trabeculated area and $\mathrm{RV} \mathrm{NC/C}$ ratio along the whole $\mathrm{RV}$ free wall. In addition, reverberation artifacts as well as near field artifacts accounted for an inadequate quantification of RV trabeculation.

The data comparing RV parameters in LVNC and controls are summarized in Tables 2 and 3. RV size was assessed by RV ED area and RV ED basal diameter, while RV systolic function was determined by FAC. All these parameters were comparable in LVNC and controls, and this finding was observed with both imaging modalities (Fig. 2). In contrast, RV ED trabeculated area in $4 \mathrm{CH}$ view and RV ED trabeculated volume were significantly higher in LVNC as compared to controls (Table 3, Fig. 3A, B). There was a major overlap of values obtained in patients and controls. Only $6(30 \%)$ patients displayed values above the upper limit of normal for both parameters (mean $+2 \mathrm{SD}$; $12.4 \mathrm{~cm}^{2}$ for area; $50.9 \mathrm{~mL}$ for volume). Similarly, $\mathrm{RV} \mathrm{ED} \mathrm{NC} / \mathrm{C}$ ratio in SAX and $\mathrm{RV} \mathrm{ED} \mathrm{NC/C}$ ratio in $4 \mathrm{CH}$ were significantly higher in LVNC than in controls (Table 3, Fig. 4A, B), but with a major overlap between the two groups. Six (30\%) patients displayed values above the upper limit of normal for NC/C in SAX (4.74), and only 4 (20\%) patients displayed values above the upper limit of normal for $\mathrm{NC} / \mathrm{C}$ in $4 \mathrm{CH}$ (4.22). RV ED NC/C ratio in SAX as well as $\mathrm{RV} \mathrm{ED} \mathrm{NC/C}$ ratio in $4 \mathrm{CH}$ correlated with LV ED NC/C ratio in long axis (SAX: $r=0.61$, $\beta=0.60, \mathrm{p}=0.0044$, Fig. $4 \mathrm{C} ; 4 \mathrm{CH}: \mathrm{r}=0.77$, 


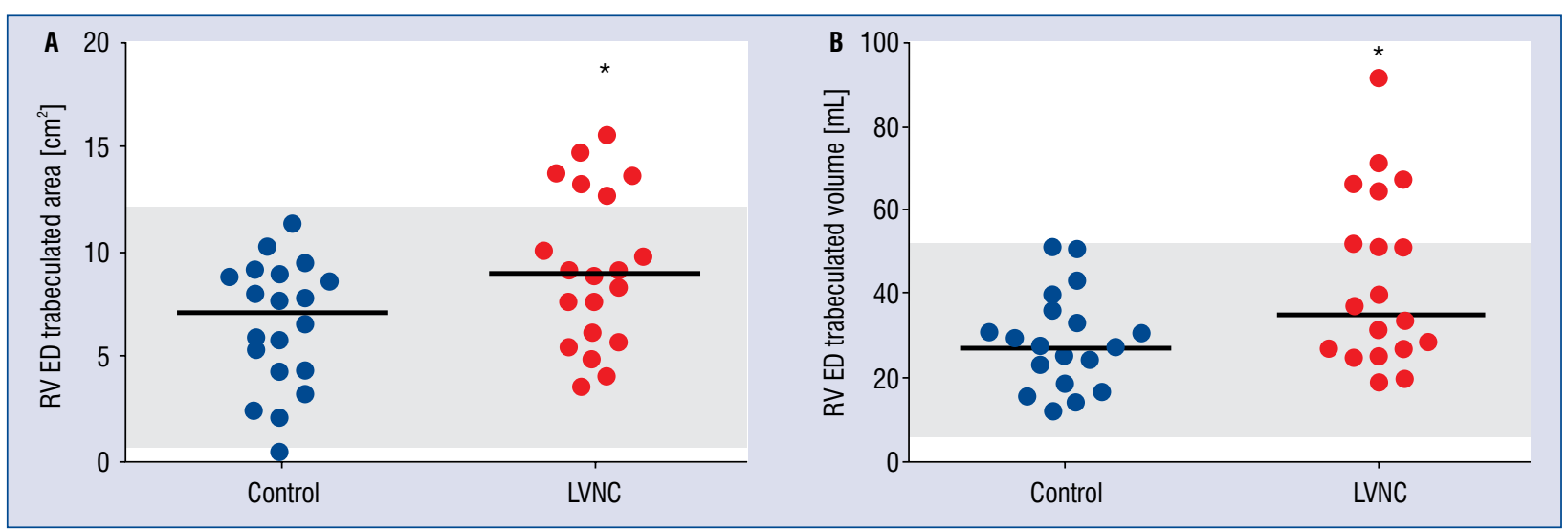

Figure 3. End-diastolic (ED) trabeculated area (A) and end-diastolic (ED) trabeculated volume (B) as assessed in cardiac magnetic resonance (CMR) in left ventricular non-compaction cardiomyopathy (LVNC) patients as compared to controls. Lines represent median values. Upper and lower limit of normal (gray): $6(30 \%)$ patients are above the upper limit for both parameters; RV - right ventricle; ${ }^{*} p<0.05$.

$\beta=0.70, \mathrm{p}=0.0001$, Fig. 4D). No significant correlation was observed for RVEF and RV ED NC/C ratio, neither in $4 \mathrm{CH}$ nor in $\mathrm{SAX}(\mathrm{r}=-0.104$, $\beta=0.21, \mathrm{p}=0.66$ ).

\section{Discussion}

This study assessed RV morphology, size, and function in LVNC patients and controls using two different imaging modalities (CMR and TTE). $\mathrm{RV}$ size and function were comparable in LVNC patients and controls by use of both modalities. Parameters indicating right ventricular involvement in LVNC such as RV ED trabeculated area and volume measured by CMR were significantly higher in the LVNC group although there was a major overlap between RV trabeculation of LVNC patients and controls hampering diagnosis of $\mathrm{RV}$ involvement in LVNC.

While several studies have compared CMR and TTE in LVNC patients regarding the LV [25, 26], this is the first study to do so for RV parameters. Assessment of RV size exhibited good correlation between the two imaging modalities. Dimensional parameters such as RV ED area and RV ED basal diameter exhibited higher values in CMR as compared to TTE. This is in line with the current guidelines reporting higher values for these parameters in CMR $[33,34]$ as compared to TTE [32]. RV systolic function assessed by FAC exhibited good correlation between the two imaging modalities. In contrast to the afore-mentioned dimensional parameters, the values for FAC were very similar with both methods presumably be- cause dimensional parameters are considered in a relative manner when a fraction such as FAC is calculated.

To assess the extent of non-compaction in the RV myocardium, different parameters were measured such as trabeculated area, trabeculated volume, and $\mathrm{NC} / \mathrm{C}$ ratio in long and short axis. While $\mathrm{RV} \mathrm{ED} \mathrm{NC/C}$ ratio in long axis has been measured in a previous study [22], the other parameters have not been investigated yet in LVNC patients. In contrast to CMR, it was not feasible to assess the extent of non-compaction in the RV myocardium by TTE for different reasons. First, there is no controllable echocardiographic equivalent to the CMR short axis with whole heart coverage to calculate RV trabeculated volume. In theory, this is feasible in a $3 \mathrm{D}$ echocardiography data set; however, current technology does not provide sufficient spatial solution to reliably assess $\mathrm{NC} / \mathrm{C}$ ratio. Second, the decline of lateral resolution with imaging depth, reverberations, and near field artifacts in combination with suboptimal acoustic windows in a subset of patients hampered the accurate quantification of $\mathrm{RV}$ trabeculated area and RV ED NC/C ratio.

Cardiac magnetic resonance imaging and TTE displayed similar RV size and function in LVNC as compared to controls. This seems to be in contrast to a previous study describing impaired RV function in LVNC [22]. In the cited study, however, LVNC patients were at a later stage of the disease as indicted by higher age, lower LVEF, and higher rate of heart failure. In line with this, studies examining $\mathrm{RV}$ function in LVNC patients revealed an associa- 


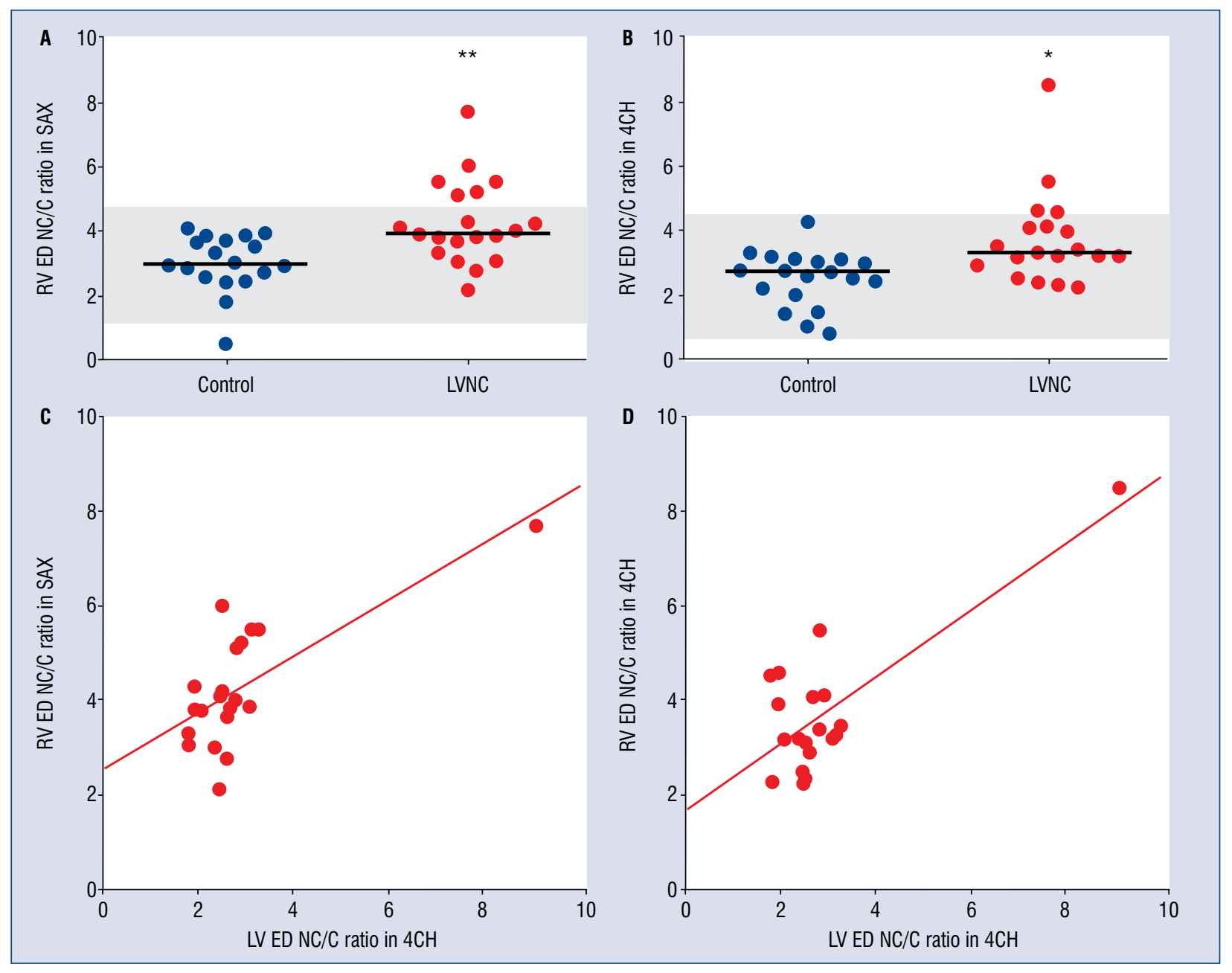

Figure 4. Right ventricular end-diastolic non-compacted to compacted (RV ED NC/C) ratio as assessed in cardiac magnetic resonance (CMR) short axis (SAX) view $(A)$ and in CMR four-chamber $(4 C H)$ view $(B)$ in left ventricular non-compaction cardiomyopathy (LVNC) patients as compared to controls. Lines represent median values. Upper and lower limit of normal (gray): 6 (30\%) patients are above the upper limit of non-compacted to compacted (NC/C) ratio in SAX, 4 (20\%) patients above the upper limit of NC/C ratio in $4 \mathrm{CH}$. RV ED NC/C ratio as assessed in CMR SAX (C) as well as when assessed in $\mathrm{CMR} 4 \mathrm{CH}$ (D) exhibits significant correlation with conventional left ventricular end-diastolic (LV ED) NC/C ratio as assessed in $\mathrm{CMR} 4 \mathrm{CH} ;{ }^{*} \mathrm{p}<0.05 ;{ }^{*} \mathrm{p}<0.01$.

tion of RV dysfunction with LV dysfunction as well as with heart failure symptoms [20,21].

To assess RV non-compaction in LVNC, the afore-mentioned novel parameters were determined. RV ED trabeculated area and RV ED trabeculated volume were higher in LVNC as compared to controls. Similarly, RV ED NC/C ratio in both long and short axis was increased in LVNC as compared to controls. However, it is questionable whether these differences represent an RV involvement since there was a major overlap with $70 \%$ of LVNC patients within the normal range. This is partially in line with another cohort of LVNC patients, where $\mathrm{RV} \mathrm{ED} \mathrm{NC} / \mathrm{C}$ ratio in long axis was not increased as compared to a control group [22].
RV ED NC/C ratio in SAX and $4 \mathrm{CH}$ correlated significantly with the extent of LV non-compaction as assessed by a standardized protocol [27]. Similar to these results, another study described that apical trabecular thickness in the RV correlates with LV end-systolic NC/C ratio [22]. To exclude that the correlation was only due to the 1 patient with much higher $\mathrm{NC} / \mathrm{C}$ ratios, the analysis was repeated without this patient. In the latter analysis, the correlation was still significant when RV ED $\mathrm{NC} / \mathrm{C}$ ratio was measured in SAX while it was not significant thereafter when measured in $4 \mathrm{CH}$. The reason for the more robust correlation with the SAX method may be related to the observation that $\mathrm{RV} \mathrm{NC} / \mathrm{C}$ SAX displays the maximal NC/C ratio of 
all RV segments (as CMR SAX covers the whole $\mathrm{RV})$. In contrast, $\mathrm{RV} \mathrm{NC/C}$ in $4 \mathrm{CH}$ only covers a small part of the RV free wall, which may not be representative for the whole RV.

The present findings suggest that (1) the RV may be affected in some patients with LVNC and that (2) RV ED NC/C ratio measured in short axis is a more reliable parameter for evaluating $\mathrm{RV}$ involvement than $\mathrm{RV} \mathrm{ED} \mathrm{NC/C}$ ratio measured in long axis. However, for all parameters of RV non-compaction determined in this study, there is a major overlap between LVNC and controls, which seems to be related to the prominent trabeculation of the RV in normal individuals, and which renders the diagnosis of RV involvement in LVNC patients very difficult. It is almost impossible to define a diagnostic cutoff value for RV trabeculation with only $30 \%$ of LVNC patients above the upper limit of normal.

Interestingly, also for LV morphology, a recent study on the current CMR criteria for the diagnosis of LVNC describes a high variability and their prognostic value seems questionable [14]. This and the present findings suggest that a more comprehensive approach including LV and RV morphology as well as genetic and functional parameters may increase diagnostic accuracy. Further studies will be needed to examine this hypothesis.

Cardiac magnetic resonance imaging and TTE provide similar quantitative data on RV size and function in LVNC patients suggesting that these parameters can be assessed by TTE in clinical routine, resulting in lower cost and avoiding problems related to implantable cardioverter-defibrillator and claustrophobia. In contrast, CMR is the method of choice for morphological assessment of RV trabeculations.

A limitation of this study is that LVNC is a very rare disease, accounting for the small number of patients. In addition, referral bias may have affected the results.

\section{Conclusions}

Some patients with LVNC may exhibit non-compaction of the RV myocardium with higher values for $\mathrm{RV}$ trabeculated area, $\mathrm{RV}$ trabeculated volume, and $\mathrm{RV} \mathrm{NC/C}$ ratio as compared to control individuals. Consistent with this, the $\mathrm{NC} / \mathrm{C}$ ratio exhibited a fair correlation between RV and LV. Nevertheless, there is substantial overlap with $\mathrm{RV}$ trabeculation in healthy individuals. Thus, quantification of RV trabeculation does not allow separation of LVNC form healthy individuals. Even though measurement of RV trabeculation may not serve as an independent diagnostic tool, it was thought, herein, that it improves the evaluation of LVNC patients. In the future it may serve as an additional parameter in comprehensive diagnostic and prognostic approaches possibly including $\mathrm{LV}$ and RV morphology as well as genetic and functional parameters.

\section{Acknowledgments}

The study was supported by the Swiss Heart Foundation.

\section{Conflict of interest: None declared}

\section{References}

1. Maron BJ, Towbin JA, Thiene G, et al. Contemporary definitions and classification of the cardiomyopathies: an American Heart Association Scientific Statement from the Council on Clinical Cardiology, Heart Failure and Transplantation Committee; Quality of Care and Outcomes Research and Functional Genomics and Translational Biology Interdisciplinary Working Groups; and Council on Epidemiology and Prevention. Circulation. 2006; 113(14): 1807-1816, doi: 10.1161/CIRCULATIONAHA.106.174287, indexed in Pubmed: 16567565.

2. Finsterer J, Stöllberger C, Towbin JA. Left ventricular noncompaction cardiomyopathy: cardiac, neuromuscular, and genetic factors. Nat Rev Cardiol. 2017; 14(4): 224-237, doi: 10.1038/ nrcardio.2016.207, indexed in Pubmed: 28079110.

3. Stöllberger C, Finsterer J. Left ventricular hypertrabeculation/ noncompaction. J Am Soc Echocardiogr. 2004; 17(1): 91-100, doi: 10.1016/s0894-7317(03)00514-5.

4. Engberding R, Bender F. Identification of a rare congenital anomaly of the myocardium by two-dimensional echocardiography: persistence of isolated myocardial sinusoids. Am J Cardiol. 1984; 53(11): 1733-1734, doi: 10.1016/0002-9149(84)90618-0, indexed in Pubmed: 6731322.

5. Jenni R, Goebel N, Tartini R, et al. Persisting myocardial sinusoids of both ventricles as an isolated anomaly: echocardiographic, angiographic, and pathologic anatomical findings. Cardiovasc Intervent Radiol. 1986; 9(3): 127-131, doi: 10.1007/BF02577920, indexed in Pubmed: 3089618.

6. Oechslin E, Jenni R. Left ventricular non-compaction revisited: a distinct phenotype with genetic heterogeneity? Eur Heart J. 2011; 32(12): 1446-1456, doi: 10.1093/eurheartj/ehq508, indexed in Pubmed: 21285074.

7. Oechslin EN, Attenhofer Jost CH, Rojas JR, et al. Long-term follow-up of 34 adults with isolated left ventricular noncompaction: a distinct cardiomyopathy with poor prognosis. J Am Coll Cardiol. 2000; 36(2): 493-500, doi: 10.1016/s0735-1097(00)00755-5, indexed in Pubmed: 10933363.

8. Stämpfli SF, Erhart L, Hagenbuch N, et al. Prognostic power of NT-proBNP in left ventricular non-compaction cardiomyopathy. Int J Cardiol. 2017; 236: 321-327, doi: 10.1016/j.ijcard.2017.02.064, indexed in Pubmed: 28268080.

9. Chin TK, Perloff JK, Williams RG, et al. Isolated noncompaction of left ventricular myocardium. A study of eight cases. Circula- 
tion. 1990; 82(2): 507-513, doi: 10.1161/01.cir.82.2.507, indexed in Pubmed: 2372897.

10. Murphy RT, Thaman R, Blanes JG, et al. Natural history and familial characteristics of isolated left ventricular non-compaction. Eur Heart J. 2005; 26(2): 187-192, doi: 10.1093/eurheartj/ehi025, indexed in Pubmed: 15618076.

11. Lofiego C, Biagini E, Pasquale F, et al. Wide spectrum of presentation and variable outcomes of isolated left ventricular non-compaction. Heart. 2007; 93(1): 65-71, doi: 10.1136/hrt.2006.088229, indexed in Pubmed: 16644854.

12. Aras D, Tufekcioglu O, Ergun K, et al. Clinical features of isolated ventricular noncompaction in adults long-term clinical course, echocardiographic properties, and predictors of left ventricular failure. J Card Fail. 2006; 12(9): 726-733, doi: 10.1016/j.cardfail.2006.08.002, indexed in Pubmed: 17174235.

13. Lilje C, Rázek V, Joyce JJ, et al. Complications of non-compaction of the left ventricular myocardium in a paediatric population: a prospective study. Eur Heart J. 2006; 27(15): 1855-1860, doi: 10.1093/eurheartj/ehl112, indexed in Pubmed: 16818458.

14. Ivanov A, Dabiesingh DS, Bhumireddy GP, et al. Prevalence and Prognostic Significance of Left Ventricular Noncompaction in Patients Referred for Cardiac Magnetic Resonance Imaging. Circ Cardiovasc Imaging. 2017; 10(9), doi: 10.1161/CIRCIMAGING.117.006174, indexed in Pubmed: 28899950.

15. Ritter M, Oechslin E, Sütsch G, et al. Isolated noncompaction of the myocardium in adults. Mayo Clin Proc. 1997; 72(1): 26-31, doi: 10.4065/72.1.26, indexed in Pubmed: 9005281.

16. Bleyl SB, Mumford BR, Brown-Harrison MC, et al. Xq28-linked noncompaction of the left ventricular myocardium: prenatal diagnosis and pathologic analysis of affected individuals. Am J Med Genet. 1997; 72(3): 257-265, indexed in Pubmed: 9332651.

17. Gebhard C, Stähli BE, Greutmann M, et al. Reduced left ventricular compacta thickness: a novel echocardiographic criterion for non-compaction cardiomyopathy. J Am Soc Echocardiogr. 2012; 25(10): 1050-1057, doi: 10.1016/j.echo.2012.07.003, indexed in Pubmed: 22883316.

18. Chiribiri A, Leuzzi S, Salvetti I, et al. Isolated noncompaction of the right ventricular myocardium in the adulthood? Int J Cardiol. 2009; 134(1): e17-e19, doi: 10.1016/j.ijcard.2008.02.001, indexed in Pubmed: 18378024.

19. Ichida F, Hamamichi Y, Miyawaki T, et al. Clinical features of isolated noncompaction of the ventricular myocardium: longterm clinical course, hemodynamic properties, and genetic background. J Am Coll Cardiol. 1999; 34(1): 233-240, doi: 10.1016/ s0735-1097(99)00170-9, indexed in Pubmed: 10400016.

20. Leung SW, Elayi CS, Charnigo RJ, et al. Clinical significance of right ventricular dysfunction in left ventricular non-compaction cardiomyopathy. Int J Cardiovasc Imaging. 2012; 28(5): 1123-1131, doi: 10.1007/s10554-011-9925-z, indexed in Pubmed: 21792620.

21. Nucifora G, Aquaro GD, Masci PG, et al. Magnetic resonance assessment of prevalence and correlates of right ventricular abnormalities in isolated left ventricular noncompaction. Am J Cardiol. 2014; 113(1): 142-146, doi: 10.1016/j.amjcard.2013.08.049, indexed in Pubmed: 24176065.

22. Stacey RB, Andersen M, Haag J, et al. Right ventricular morphology and systolic function in left ventricular noncompaction cardiomyopathy. Am J Cardiol. 2014; 113(6): 1018-1023, doi: 10.1016/j.amjcard.2013.12.008, indexed in Pubmed: 24462071.

23. Stämpfli SF, Donati TG, Hellermann J, et al. Right ventricle and outcome in left ventricular non-compaction cardiomyopathy. J Cardiol. 2020; 75(1): 20-26, doi: 10.1016/j.jjcc.2019.09.003, indexed in Pubmed: 31587941.

24. Bleeker GB, Steendijk P, Holman ER, et al. Assessing right ventricular function: the role of echocardiography and complementary technologies. Heart. 2006; 92 Suppl 1: i19-i26, doi: 10.1136/ hrt.2005.082503, indexed in Pubmed: 16543597.

25. Alhabshan F, Smallhorn JF, Golding F, et al. Extent of myocardial non-compaction: comparison between MRI and echocardiographic evaluation. Pediatr Radiol. 2005; 35(11): 1147-1151, doi: 10.1007/s00247-005-1551-2, indexed in Pubmed: 16086159.

26. Thuny F, Jacquier A, Jop B, et al. Assessment of left ventricular non-compaction in adults: side-by-side comparison of cardiac magnetic resonance imaging with echocardiography. Arch Cardiovasc Dis. 2010; 103(3): 150-159, doi: 10.1016/j.acvd.2010.01.002, indexed in Pubmed: 20417446.

27. Petersen SE, Selvanayagam JB, Wiesmann F, et al. Left ventricular non-compaction: insights from cardiovascular magnetic resonance imaging. J Am Coll Cardiol. 2005; 46(1): 101-105, doi: 10.1016/j.jacc.2005.03.045, indexed in Pubmed: 15992642.

28. Jacquier A, Thuny F, Jop B, et al. Measurement of trabeculated left ventricular mass using cardiac magnetic resonance imaging in the diagnosis of left ventricular non-compaction. Eur Heart J. 2010; 31(9): 1098-1104, doi: 10.1093/eurheartj/ehp595, indexed in Pubmed: 20089517.

29. Kramer CM, Barkhausen J, Flamm SD, et al. Standardized cardiovascular magnetic resonance imaging (CMR) protocols, society for cardiovascular magnetic resonance: board of trustees task force on standardized protocols. J Cardiovasc Magn Reson. 2008; 10: 35, doi: 10.1186/1532-429X-10-35, indexed in Pubmed: 18605997.

30. Kramer CM, Barkhausen J, Flamm SD, et al. Standardized cardiovascular magnetic resonance (CMR) protocols 2013 update. J Cardiovasc Magn Reson. 2013; 15: 91, doi: 10.1186/1532-429X15-91, indexed in Pubmed: 24103764.

31. Rudski L, Lai W, Afilalo J, et al. Guidelines for the Echocardiographic Assessment of the Right Heart in Adults: A Report from the American Society of Echocardiography. J Am Soc Echocardiogr. 2010; 23(7): 685-713, doi: 10.1016/j.echo.2010.05.010.

32. Lang R, Badano L, Mor-Avi V, et al. Recommendations for Cardiac Chamber Quantification by Echocardiography in Adults: An Update from the American Society of Echocardiography and the European Association of Cardiovascular Imaging. Eur Heart J Cardiovasc Imaging. 2015; 16(3): 233-271, doi: 10.1093/ehjci/jev014.

33. Maceira AM, Prasad SK, Khan M, et al. Reference right ventricular systolic and diastolic function normalized to age, gender and body surface area from steady-state free precession cardiovascular magnetic resonance. Eur Heart J. 2006; 27(23): 2879-2888, doi: 10.1093/eurheartj/ehl336, indexed in Pubmed: 17088316.

34. Le Ven F, Bibeau K, De Larochellière É, et al. Cardiac morphology and function reference values derived from a large subset of healthy young Caucasian adults by magnetic resonance imaging. Eur Heart J Cardiovasc Imaging. 2016; 17(9): 981-990, doi: 10.1093/ehjci/jev217, indexed in Pubmed: 26354980. 